\title{
EL DISEÑO DE LA PROPIA ESTRATEGIA PROFESIONAL DESIGN OF THE OWN PROFESSIONAL STRATEGY
}

\author{
AnTONio VALls Roig \\ Consultor de Desarrollo Directivo de HUMAN SUCCES \\ Valls.antonio@gmail.com
}

\section{Resumen}

El presente artículo trata de destacar los cuatro puntos clave que un profesional debe recorrer constantemente a lo largo de su vida laboral, si realmente quiere considerarse «gestor» de su trayectoria profesional. Finalmente, tu carrera «la gestionas», o «te la gestionan».

Con especial énfasis en los postulados de Daniel Levinson sobre las estaciones de vida, el artículo destaca no obstante la necesidad de estar sensibilizado por la propia carrera, de ponerse objetivos, de planificar y actuar.

\section{Palabras clave}

Trayectoria profesional, objetivos, plan, contactos, estaciones, diseño, estrategia

\begin{abstract}
In this article, the author wants to show the four key points importance, to take into account when thinking on managing your career. Two options are at hand: either "you» are the manager, or the ones who will manage your career will be «the others».

Emphasizing Daniel Levinson's opinions and research regarding life seasons, this article shows -nevertheless- the need to be sensitive at all times -as far as your career is concerned-, and the need to have goals, make plans, and do something, like in a business environment.
\end{abstract}

\section{Key Words}

Professional trajectory, objects, plan, contacts, deisign, strategy 
La prestigiosa revista Fortune publicó en 1994, hace más de una década, un concienzudo estudio relativo al mundo del trabajo del futuro, en el que se destacan sus tres principales características (que hoy por hoy no han hecho más que acentuarse):

$1^{\circ}$ Para el empleado, cada vez será más difícil encontrar trabajo, porque los puestos disponibles serán pocos, y las solicitudes para cubrirlos muchas. Para un candidato a un determinado empleo existirá la permanente amenaza de que se ofrezca otro competidor mejor formado, y dispuesto a trabajar más horas... por menos sueldo del que le gustaría percibir.

$2^{\circ}$ Las empresas no podrán soportar la carga económica que supone pagar altas retribuciones a empleados que dedican ocho horas diarias a la empresa. Para los trabajos que precisen tal inversión en tiempo, se preferirá contratar colaboradores o ayudantes a tiempo parcial.

$3^{\circ}$ Los puestos de responsabilidad de la empresa se convertirán en puestos de alto o altísimo riesgo de pérdida de empleo. Tradicionalmente era más incierto e inseguro, ejercer una profesión liberal que un puesto de dirección en la empresa privada. Hoy, quizás esto ya no es así, y lo será todavía menos en el futuro. Este tercer punto es producto de la alta especialización que conllevan determinados cargos.

Puntos, todos ellos, que exigen más de la persona, en la gestión de su trayectoria profesional.

\section{¿Porqué necesitamos una Estrategia Profesional?}

La persona que ocupa un puesto de trabajo y no da los pasos necesarios para dirigirse hacia otro "mejor», es porque quizás no quiere, y/o no sabe. Los motores de la acción humana eficaz son ambos: «querer ir» $\mathrm{y}$ "saber ir» (Hersey, Blanchard, y Johnson, $2007-1^{\text {a }}$ publicación, 1972)

Las oportunidades y posibilidades de cambio -lo admitamos o no- están ahí para todo el mundo. Pero las más de las veces nos abruma la idea de alimentar y -aun más- perseguir nuestros sueños. En nuestra vida profesional se da una paradoja: nuestro trabajo procuramos hacerlo bien; pero nuestra vida profesional, ¿hacia dónde va?. Y entonces, cuando surge un imprevisto en nuestra carrera, no sabemos donde encajarlo, y quedamos envueltos en un mar de dudas. Y los imprevistos -cambios, despidos, obstáculos...- están aquí para quedarse (Rifkin, 1996)

La mejor manera de encarar un imprevisto es generarse previamente un plan «B» (Fisher y Ury, 1996). Tener un plan «B» es tener una contestación lo bastante clara y concisa a la pregunta: ¿Qué haré cuando por las razones que sea, ya no tenga el empleo que tengo hoy? ¿Cómo me ganaré la vida? ¿Dónde y cómo ejerceré mi profesión actual o una diferente?. De algún modo, hay que anticiparse a los acontecimientos. Hay que prepararse. Pues como dicen los escritos sobre el arte de la guerra chino, «si te pones en un lugar en que no te puedan derrotar, serás un buen general» (Sun Tzu, 2006).

En el terreno del empleo, ello implica trabajar en tres frentes o factores de éxito profesional:

- El de los propios objetivos profesionales, a corto, medio y largo plazo.

- El del estudio e investigación del terreno, para tener claro el mapa que nos permitirá movernos desde nuestra posición actual, a la que corresponde a nuestro siguiente objetivo.

- El de la gestión de nuestra red personal de contactos.

Mucho es lo que se ha dicho y escrito sobre fijación de objetivos. Pero en el plano de la propia trayectoria profesional, resulta altamente iluminador contemplar el problema desde la perspectiva de las estaciones de la vida.

\section{Las estaciones de la vida}

Para situar nuestras inquietudes y ambiciones profesionales de hoy en un contexto vital que sea apropiado, es interesante analizar las conclusiones de Daniel Levinson (1978), fruto de la observación de la trayectoria de diversos profesionales de diferentes campos. 
Levinson observó que la vida profesional, se desarrolla en una especie de sucesivas mareas marcadas por etapas de transición, que tienen lugar aproximadamente cada diez años. Se afianzó también en la idea de que entre los cuarenta y los cuarenta y cinco años de edad, la persona suele pasar por una crisis de virulencia igual o mayor que la de la pubertad y la adolescencia. Es la crisis de la «media edad». El profesor Rodríguez Porras (1995) ha hecho asimismo interesantes aportaciones a este tema, en especial referentes a las últimas etapas de la vida.

Levinson detecta las siguientes peculiaridades en la vida de la persona, según la edad en la que está:

\section{Entre los 20 y los 27 años de edad}

A partir de los 20, suele terminar la crisis de la adolescencia, -una crisis de crecimiento y de identidad- y se produce la entrada del individuo en el mundo adulto. Las elecciones que se hacen en esta etapa de la vida son muy importantes, y suelen causar inquietudes y honda ansiedad. Estas decisiones comportan la elección de profesión, y la elección de pareja. Puede también darse la necesidad de buscar e identificar a un mentor, que guíe los inicios de la vida profesional del individuo. Este mentor puede ser una sola persona o un grupo de personas, o puede hacer la función de mentor una «empresa escuela» o empresa líder, en que todo se hace buscando la excelencia, para garantizar la futura competitividad.

Todo este proceso de selección entre opciones muy diversas está presidido por la paulatina concepción de un "sueño», relativo a la manera como se proyecta vivir la vida personal y profesional en el futuro. Se trata de un sueño que comprende el propio proyecto existencial.

En esta primera etapa la persona puede asumir un serio compromiso con sus propias opciones, o decidir a la ligera y sin profundizar. Este segundo caso suele acarrear problemas más tarde, de los que se resentirá su trayectoria profesional.

\section{Entre los 28 y los 32 años de edad:}

Este período es de crisis y transición hacia la siguiente etapa, y se caracteriza por el sentido de urgencia que invade a la persona, al adquirir conciencia del paso de los años. Puede conllevar ajustes en la decisiones vitales fundamentales del pasado, con el objeto de intentar superar las limitaciones personales y profesionales que ahora, ya un poco mayor, percibe.

\section{Entre los 33 y los 40 años de edad:}

Recuperada la calma, en este intervalo se avanza con tendencia a ser cada vez más uno mismo, y a afianzarse en lo que se está haciendo. Es una etapa de asentamiento en la que cobra importancia el echar raíces, si ello no se ha hecho ya.

\section{Entre los 41 y 45 años de edad:}

En este momento es cuando se suele entrar en la mencionada crisis de la «media edad». Varios factores nos inducen a ella. El más importante es quizás que a esta edad se hace perentoria la necesidad de revisar el proyecto existencial que fue concebido en nuestra entrada en el mundo adulto. Las más de las veces, el paso del tiempo se ha encargado de invalidar una buena parte del mismo.

En lo referente al matrimonio, con suerte sólo será necesario hacer una pequeña actualización. Pero en transiciones turbulentas puede llegarse a lamentables situaciones de separación y divorcio. Por otra parte, la avanzada edad de nuestros progenitores, nos hace percibir con más realismo nuestra propia finitud. Esto nos obliga a asumir la edad que tenemos, y a desear concentrarnos en intereses todavía más propios y genuinos.

En contrapartida, tras esta crítica transición nos aceptamos mejor a nosotros mismos tal como somos, y salimos reforzados en lo que a auto conocimiento y auto aceptación se refiere. Curiosamente con el transcurso de la edad se nos despierta un incipiente deseo de convertirnos nosotros mismos en mentores de otras personas. 
En lo que concierne a nuestra propia trayectoria profesional, en esta edad formulamos una profunda revisión, que suele desembocar en alguna de las tres posibilidades siguientes: continuidad de la situación vigente, ruptura-declinación e inestabilidad, avance a partir de un nuevo cambio. La primera y la tercera suelen ser las más afortunadas, y la primera la que se da con más frecuencia.

\section{Entre los 46 y los 50 años de edad:}

Esta edad coincide con la entrada a adulto mayor, y en ella se suelen efectuar pequeños cambios de acomodo al nuevo ritmo vital que pide el ser humano en esta estación. Se trata de casi imperceptibles variaciones que se imprimen en la vida familiar y de trabajo.

\section{A partir de los 51 años de edad:}

Es en este período en el que al principio se vive una nueva revisión de la propia trayectoria, pero en el que enseguida se entra en una fase de plenitud que durará ya el resto de la vida hasta su culminación, momento que puede tener lugar alrededor de los sesenta y pico de años. Para entonces la declinación física aparece ya de modo patente. En esta transición a la vejez -por así llamarla-, se advierten en ocasiones unos muy notables rebrotes de creatividad.

La confrontación de la situación actual del lector con este breve resumen, en que se sintetiza -de acuerdo a los mencionados investigadores- lo que acostumbra a ser importante para el ser humano en cada fase de la vida, puede avivar una reflexión muy positiva para la redirección de la propia trayectoria profesional.

Pero no hay que descuidar el hecho -como nos indica Ben Ball (1996)- de que un estudio como el que acabamos de repasar puede reflejar en algunos aspectos el entorno económico y social en que vivieron los investigados, diferente del que estamos viviendo en la actualidad. Sin embargo como seres humanos que somos, nuestras inclinaciones esenciales son siempre las mismas, no importa la época y el lugar en que estemos: siempre necesitaremos "vivir, amar, aprender y dejar un legado» (Covey, 1994). Pero nuestras respuestas pueden ser distintas según la cultura en que nos hallemos inmersos, y las expectativas que ello nos genere.

CuAdro 1. Las estaciones de la vida.

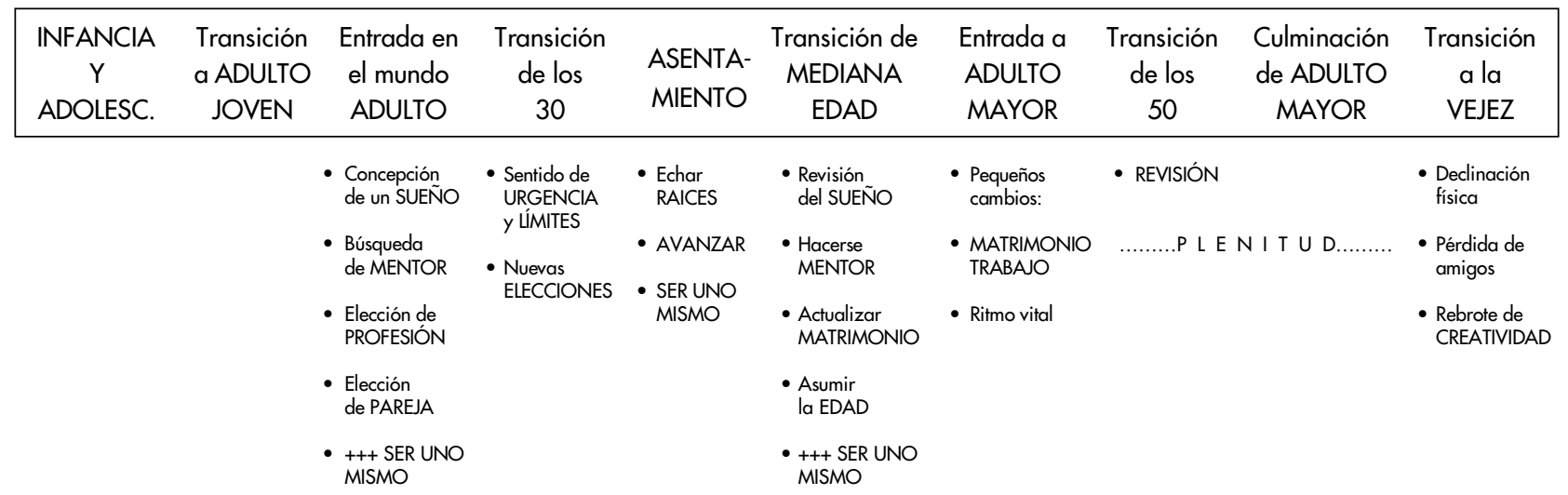

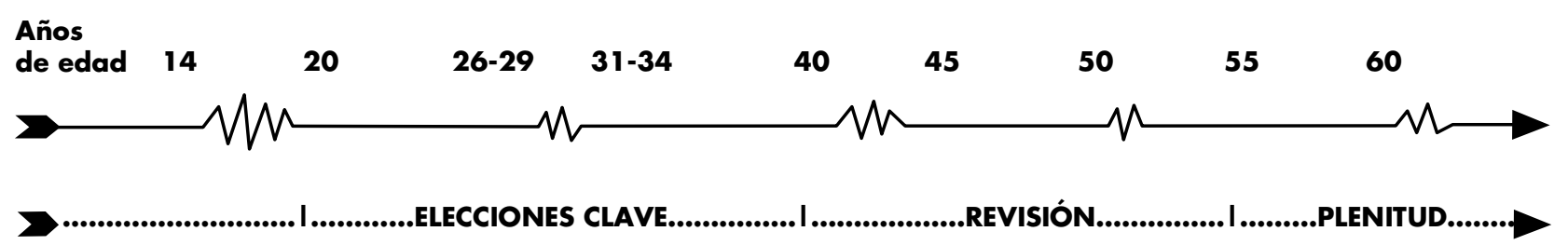




\section{Marcarse objetivos profesionales valiosos}

Ello implica:

- Llegar a una idea razonablemente realista de quién es uno mismo, y de la etapa de la vida en que uno se encuentra.

- Adquirir un sentido de "misión», de destino, de cuál es nuestro papel en este mundo.

- Decidirse a recorrer una trayectoria profesional, sobre la base de nuestras mejores aptitudes, y habilidades.

Para ponerse objetivos que tengan sentido en nuestra vida profesional, hay que afrontar dos tareas básicas: la predefinición de objetivos, y la obtención de una clara conciencia del «mapa» que se percibe en torno a cada objetivo.

En este proceso la dificultad mayor suele ser nuestro poco conocimiento de dicho «mapa». La extensión de nuestros deseos acostumbra a ser muy superior a la de nuestros conocimientos sobre la realidad a la que enfocamos nuestras querencias.

Deben darse pues algunas condiciones:

- Realismo.

- Persistir en los objetivos que nos marquemos.

- Coherencia con las propias capacidades.

- Asumir que no hay vuelta atrás.

Y por supuesto que para que un objetivo sea válido, tiene que gozar de algunos atributos:

* Debe ser medible y observable.

* Debe ser entendible.

* Debe ser adecuado en el tiempo.

* Debe ser alcanzable y asequible.

* Debe ser posible seguirlo y controlarlo.

\section{Caso Florencio}

"Tengo 48 años y estudié Ciencias Empresariales en la $U A B$. He cosechado además una amplia experiencia profesional en el mundo de la empresa».
"Durante diez años trabajé en BELTEX, una empresa familiar en la que mi padre tenía el $36 \%$ de la acciones y mi tío un tercio. Mi padre se desempeñaba como gerente. Pero luego dejé el empleo».

"Descubrí que BELTEX sólo tenía razón de ser como subsidiaria de una acería, pero no consegui que nadie nos comprara la empresa. También me desvinculó el hecho de que en un momento dado mi padre no participó en una ampliación de capital, lo que le dejó con un patrimonio muy pequeño en la compañía. Y finalmente, la incorporación en la organización de algunos familiares pertenecientes al círculo del socio mayoritario precipitó mi decisión».

«Para no perjudicar a BELTEX, no empecé a buscar otro trabajo hasta después de dimitir, y esto tal vez fue un error. En nueve meses no tuve nada que hacer salvo un pequeño trabajo de consultoría. Después entré como Director Comercial en una empresa que hizo suspensión de pagos al cabo de un año».

"Con cierta facilidad encuentro a continuación un trabajo como responsable comercial y de marketing de "BARUNIO", en la que no me siento a gusto, y al cabo de un año entro en la consultoría Peter Townsend, en la que durante cuatro años acumulé muchas y muy variadas experiencias, hasta que entró un Consejero Delegado que desde mi punto de vista era un impresentable».

"Alentado por éxitos anteriores constituí mi propia consultoría, FLORENCIO CONSULTANTS. Los primeros años fueron un éxito, pero después, los contactos y la situación económica general no dieron más de si, y a los cuatro años abandoné el tema, para desempeñarme durante un tiempo como Director General de una empresa ex-cliente».

"Ahora hace cuatro meses que estoy buscando trabajo. En mi última empresa, la situación de dificultad económico-financiera desembocó en un cambio de todo el equipo de gestión».

"Mis relaciones con los cazatalentos han sido agradables y constructivas; pero desde un punto de vista práctico no han producido nada concreto. Muchos se quejan de que tienen poco trabajo últimamente. O a lo mejor el mío no es un curriculum tipo». 
"Estoy buscando volver a trabajar en una empresa o en una consultoría. Estoy convencido de que el tema costará pero saldré. Lo que si es importante es hacer un ejercicio de estar dispuesto a jugar y a tener una actitud positiva».

"Sigo haciendo contactos y procuro no desanimarme... y si me desanimo me lo permito solamente hasta el día siguiente por la mañana. La gente te quiere ayudar pero no es fácil: no saben como hacerlo. Estoy absolutamente abierto a esquemas diferentes; desde el esquema clásico, cada vez menos probable, de un empleo de alta dirección, hasta otro de participar como socio de un proyecto en marcha».

Estudiemos detenidamente el caso de Florencio. Quizás fue una persona en cierto modo consciente de la importancia de marcarse objetivos coherentes con sus intereses y su momento vital... Se le ve un individuo con un rumbo personal y profesional en una época muy definido, que el mismo se trabajó, analizó y elaboró. Sin embargo en una etapa posterior, su trayectoria profesional ha quedado a la deriva. Las claves podrían ser las siguientes:

a) En cuanto abandona el núcleo familiar, pierde su sensibilización con respecto al momento en que se encuentra en su trayectoria profesional. Parece poseer objetivos claros a corto, pero a medio y largo plazo opera a ciegas. No tener los objetivos claros le deja sin poder para evaluar sus propias posibilidades en cada momento del futuro, le lleva -tal vez- a sobrevalorar su situación, y principalmente, a no generarse alternativas. No dispone de un plan " $\mathrm{B}$ » en ningún momento, $\mathrm{y}$ ello le hace perder poder de direccionamiento... No es coherente con la máxima del Sun Tzu: «Si te pones en un lugar en que no te puedan derrotar, serán un buen general», que es probablemente el punto más importante a tener en cuenta, en la gestión de la propia trayectoria profesional.

b) Pero obviamente: sin sensibilización y sin plan, ni que decirse tiene que es imposible llegar a generarse un mapa (un detalle del recorrido a efectuar para llegar de «A hasta «B»), y finalmente: c) En la gestión de la red personal de contactos, Florencio camina de modo aleatorio.

En conclusión: ser consciente de que en la vida laboral hay unas estaciones más o menos previsibles, no es suficiente si no se hace algo al respecto. Gestionar la propia trayectoria profesional es, como se ha dicho más arriba, planificar y actuar. Pero hacer lo uno sin hacer lo otro es la fórmula más segura para ir a bastante menos de la mitad de la velocidad de crucero, a la que un profesional podría ir en su trayectoria profesional.

\section{Conclusión}

El desarrollo de la propia estrategia profesional consiste en repetir varias veces a lo largo de la vida, un ciclo cuyas tres etapas fundamentales son las siguientes:

\section{ELECCION -confirmación- DE DIREC- CION O RUMBO.}

2. PLANIFICACION y búsqueda de alternativas: Plan «B».

\section{ACCION.}

Cubrir las etapas uno y dos de este ciclo entraña un trabajo intelectual, mientras que para la tercera lo que se requiere es actuar. Y si no se actúa, el trabajo realizado para recorrer las etapas uno y dos no sirve.

Poner en marcha nuestra propia estrategia personal y profesional significa arrancar en un camino que nos dirige -o no- hacia un nuevo trabajo, ya sea como empleados o en un negocio propio.

\section{Bibliografía}

Ball, B. (1996). Assesing your career: time for change?. BPS books, Leicester,

Covey, S.R. (1995). Los 7 hábitos de la gente altamente efectiva. Ediciones Paidós Ibérica S.A., Barcelona

De Bono, E. (1995). El pensamiento creativo. Ediciones Paidós Ibérica Barcelona,

Fortune (1994). Seven rules to break in a de-jobbed world. Nueva York, 19 de Septiembre.

Fisher, R, Ury, W. y Patton, B. (1996). Obtenga el SI. Ediciones Gestión 2000. Barcelona 
Galvany, A. (2006). Sun Tzu, el arte de la guerra. Traducción directa del chino antiguo a cargo de Albert Galvany, coautor de la primera traducción directa y completa del chino al castellano. Incluye texto original chino; Editorial Trotta: Madrid, 2001 [ $5^{a}$ edición 2006].

Hersey, P., Blanchard, K.H, y Johnson, D.E (2007). Management of Organizational Behavior. Leading Human Resources», Prentice Hall, 2007

Levinson, D. J. (1978). The seasons of a man's life. Ballantine Books, New York,
Lladó, J.-Valls, A (1997). Consiga el Éxito en su trayectoria profesional. Ediciones Gestión 2000, S.A., Barcelona

Rifkin, J. (1996). El fin del trabajo. Ediciones Paidós Ibérica. Barcelona,

Rodríguez Porras, José $\mathrm{M}^{\mathrm{a}}$ (1995). El factor humano en la empresa. EUNSA, Pamplona

Valls Roig, A. (1998). Las 12 Habilidades Directivas clave. Gestión 2000

Valls Roig, A. (2001). Negocie, disfrute y Gane. Amat Editorial. Barcelona. 